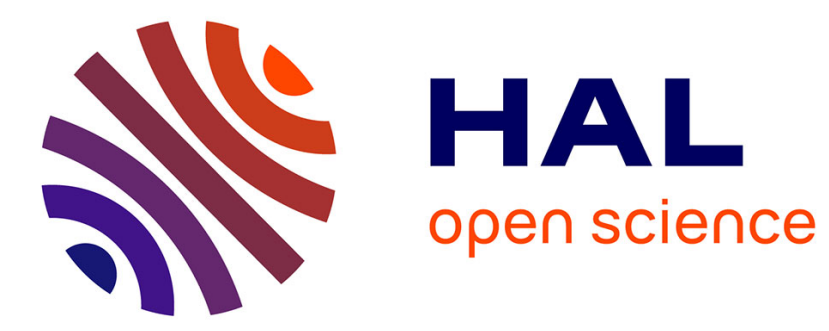

\title{
A low latency MAC Scheme for Event-Driven Wireless sensor networks.
}

Hung-Cuong Le, Hervé Guyennet, Violeta Felea, Noureddine Zerhouni

\section{To cite this version:}

Hung-Cuong Le, Hervé Guyennet, Violeta Felea, Noureddine Zerhouni. A low latency MAC Scheme for Event-Driven Wireless sensor networks.. Lecture Notes in Computer Science, 2007, 4864, pp.291301. 10.1007/978-3-540-77024-4 . hal-00400406

\section{HAL Id: hal-00400406 https://hal.science/hal-00400406}

Submitted on 30 Jun 2009

HAL is a multi-disciplinary open access archive for the deposit and dissemination of scientific research documents, whether they are published or not. The documents may come from teaching and research institutions in France or abroad, or from public or private research centers.
L'archive ouverte pluridisciplinaire HAL, est destinée au dépôt et à la diffusion de documents scientifiques de niveau recherche, publiés ou non, émanant des établissements d'enseignement et de recherche français ou étrangers, des laboratoires publics ou privés. 


\title{
A Low Latency MAC scheme for Event-Driven Wireless Sensor Networks
}

\author{
Hung-Cuong LE ${ }^{1}$, Hervé GUYENNET ${ }^{1}$, Violeta FELEA ${ }^{1}$, and Noureddine \\ ZERHOUNI $^{2}$ \\ ${ }^{1}$ Laboratoire d'Informatique de l'Université de Franche Comté, France \\ ${ }^{2}$ Laboratoire d'Automatique de Besançon, France \\ $\{$ le, guyennet, felea\}@lifc.univ-fcomte.fr \\ noureddine.zerhouni@ens2m.fr
}

\begin{abstract}
In this paper, we present a low latency media access control scheme which we call LLMAC (Low Latency MAC) for event-driven wireless sensor networks (WSN). In this kind of WSN, sensors do not regularly send data to the sink. They send a burst data only when there is an event in the monitoring area. It takes time for this burst data to arrive to the sink. Normally, these events are critical and we hope to obtain the information on the event in the shortest delay. Hence, the latency is considered to be a crucial requirement in event-driven WSN contrary to the traditional wireless networks where the fairness is the most important requirement. Our proposal LLMAC makes a trade-off between fairness and latency in order to offer a shorter latency transmission when certain events happen. The performance evaluation shows that our proposal reduces the latency in comparison to existing MAC protocols.
\end{abstract}

\section{Introduction}

Wireless sensor network is a very hot research topic tendency in distributed systems. A WSN is a network composed of hundreds to thousands of communicating sensors deployed on an area in order to collect environment events. WSN have a wide range of domain application: industry, medical, military, civilization etc. Generally, there are three models of WSN: continuous, on-demand and event-driven. In continuous WSN, sensors send data periodically to the sink. There are always sensors in the network which initiate the communication. In the on-demand WSN model, sensors send data only when they receive a request from the access point. Without request, sensors sense information and store it in their local memory. In the context of this paper, we are interested in the last model: event-driven WSN. In this model, the sensors send data only when certain events occur. For example, a wireless sensor network is deployed on a machine in a factory to detect abnormal symptoms of the machine. Sensors can sense the temperature, the vibration or the humidity of the machine. Normally, when the machine works well, sensors stay silent. When there is a problem in the machine, this problem will produce environment change: an increase in temperature, humidity of the machine or the machine vibrates faster. This ambient information can be detected by sensors and they will activate many camera or sound 
sensors to take pictures or record sounds and immediately report an event, composed of many packets, to the sink via multi-hop transmission.

In traditional wireless networks, every node is fair in term of channel access. They have the same role in the network. The transmission objective is that when a node has a data to send, it can win the channel in the shortest delay. Hence, almost every existing MAC protocol wants to guarantee the same channel access probability to every node. However, in an event-driven WSN, a communication is often multi-hop and uni-direction from nodes to the sink. An event-driven WSN is often organized in tree topology where each node establishes a data gather tree to send data to the sink. In a tree topology, sensors do not have the same role. Nodes at the leaf do not have to route data. They only have to send data when they detect an event. Nodes in the inner of the network topology have to do two tasks: sense events and route data for other nodes. Hence, in event-driven WSN, sensors are not fair and we need to design a different MAC protocol which is specialized for this kind of networks.

In a WSN, sensors are often spatially correlated. When an event happens, many sensors try to send data to the sink via gateway nodes simultaneously. If every node can transmit data at the same time, there will be no problems. However, in an interfering zone, there is only one node to transmit and all the others have to keep silent. The MAC protocol often guarantees fairness in one-hop communication. Hence, it guarantees that one node can send its packet within the shortest delay in one hop. However, a transmission delay in multi-hop communication is the elapsed time between the moments the packet started to be transmitted and the moment it reaches the sink. Hence, we need to facilitate the channel access of nodes which are nearer to the sink in order to finish the multi-hop transmission within the shortest delay.

In section 2, we present related works of MAC layer of WSNs. Then, we show a simple transmission scenario using IEEE 802.11 where the latency could be a crucial problem. In section 4, we describe LLMAC in order to reduce transmission latency for wireless sensor networks. Next, we show the effectiveness of our approach in comparison with other existing works. Finally, in section 6, we conclude and present several perspectives of our work.

\section{Related Works}

Today, research on medium access control (MAC) of wireless sensor networks is very fertile. There is a clear attempt to improve MAC protocol management of communication time between sensors, which consumes the most energy. Based on various characteristics, MAC protocol is classified into two different types: Contention-Free and Contention-Based.

Contention-free $M A C$ is based on reservation and scheduling. Here, each node announces a time slot that it wants to use to the coordinator of the network. This coordinator schedules requests and allocates each node its respective time slot. In this way, a node can access the channel without colliding with others because it is the only node which can transmit during its time slot. Bluetooth [1], TRAMA [2] and LEACH [3] are examples of this type of MAC. This technique guarantees low energy consumption because each node in the network works only during its time slot 
therefore no collisions. However, the major disadvantage of this technique is that it is not well adaptable to topology changes and is therefore non-scalable. Any insertion or suppression of a node implies a time slot reallocation for all nodes in the group. All contention-free MAC protocols for WSN are designed to support low energy consumption. Hence, they do not take the multi-hop latency into account.

Unlike this technique, contention-based $M A C$ is a protocol where every node accesses the channel in competition. Before transmitting a message, a node listens to the channel to see whether there is already a transmission in the medium. If the channel is busy, it waits for a random time and retries to check out the channel later. If the channel is free, it transmits the message.

The most well-known example of this technique is the IEEE 802.11 [4] for wireless LAN network. Indeed, this technique works well in communication between personal computers or pocket PCs, where energy consumption is not a critical problem. However, in a sensor network, the devices are small and very sensitive to energy consumption. Therefore, the MAC technique of IEEE 802.11 is not suitable for sensor networks.

After IEEE 802.11, many research projects have been carried out to optimize the existing MAC protocols to better adapt them to sensor networks. S-MAC [5] is considered to be the first MAC protocol proposal for sensor networks which tries to reduce energy consumption. In S-MAC, nodes are periodically set in listen and sleep mode, where the listen time is approximately $10 \%$ of the sleep time. In sleep mode, sensors switch off the radio to save energy. Hence, they can save up to $90 \%$ of energy compared to the normal protocols where nodes always stay active. Sensors synchronize their communication during the listen period. If a node does not have any messages to send, it switches its radio off during the sleep mode. On the contrary, it switches its radio on to transmit or receive messages. During listen time, sensors access the channel using the carrier sense multiple access with collision avoidance method (CSMA/CA) [6].

T-MAC [7] extends S-MAC by changing dynamically the listening time between two active periods. T-MAC also reduces the inactive time of the sensors compared to S-MAC. Hence, it is more energy efficient than S-MAC.

$\mathrm{B}-\mathrm{MAC}$ [8] is a modular and flexible channel access method. The objective of BMAC is to reduce the idle time of the sensors. Like S-MAC and T-MAC, nodes in BMAC switch their radio on and off periodically. However, there is no synchronization between sensors. In order for nodes to communicate, packets are sent with a longer preamble than the idle time of sensors.

These MAC protocols are designed for normal WSN where the latency is not a crucial problem. Hence, they do not take the latency into account in their proposal. To the best of our knowledge, SIFT [9] is the first MAC protocol which is designed for event-driven WSN. The main objective of SIFT is to reduce latency in the monitoring application where there are many simultaneous communications. They argue that sensors are spatially correlated. Hence, it is enough for the sink to receive fewer packets for each event. SIFT guarantees successful transmission of $\mathrm{R}$ out of $\mathrm{N}$ packet (each sensor detects an event and sends one packet) with the shortest delay where $\mathrm{N}$ is the number of nodes which detected the event. Based on the same principle, $\mathrm{CSMA} / \mathrm{p}^{*}$ [10] is proposed where $\mathrm{p}^{*}$ is also a non-uniform distribution in order to minimize latency. However, the main objective of this proposal is to reduce the 
collision but not to reduce the transmission latency for event-driven wireless sensor networks. Moreover, the proposals of SIFT and CSMA/p are applied only for one-hop WSN and each event is composed of one packet. They do not evaluate their propositions in multi-hop scenario and multi-packets per event which is very frequent in an event-driven WSN.

In order to obtain a short latency in certain data transmission scenario, several existing works have been proposed in the domain of QoS in wireless network [11, 12, 13]. The main idea of these proposals is to divide the data flow in different priority levels. By changing the value of the contention window of each priority level, they can favor high priority packets. Hence, high priority packets are sent with the shorter delay than others. Depending on each type of services, packets are assigned a certain priority level. Here, we refer to the differentiated services architecture. These models can be applied only when we know the important level of each type of packets. In case of event-driven WSN, every event is critical and needs to be transmitted to the sink within the shortest delay. We cannot always classify whether one event are more important than the others. Hence, these models cannot be applied directly in eventdriven WSN.

\section{A Transmission Scenario Using IEEE 802.11}

In this section, we present a simple transmission scenario using IEEE 802.11. We will show that IEEE 802.11 can cause latency problems for event-driven WSN.

\subsection{The IEEE 802.11 standard}

In this section, we briefly describe the MAC protocol of IEEE 802.11. Fig. 1 illustrates the channel timing of nodes in the network. In fact, the IEEE 802.11 standard proposes two modes: DCF (Distributed Coordinated Function) and PCF (Point Coordination Function). In DCF mode, there is no centralized control. Nodes use CSMA/CA protocol to access the channel in a fully distributed manner. On the contrary, in PCF mode, the base station controls the channel access of all nodes in the network. The MAC protocol is managed locally at the base station. The DCF and PCF mode can work together using different inter-frame spacing value (Fig. 1).

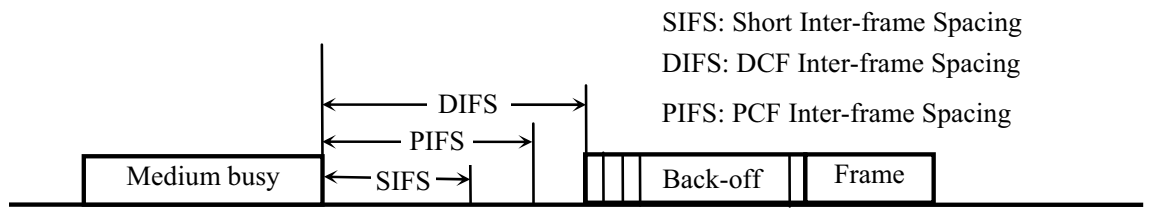

Fig. 1. Inter-frame spacing in 802.11

In order to transmit packets, nodes have to wait for a certain time: an inter-frame spacing time and a back-off time (Fig. 1). The IEEE 802.11 standard uses different inter-frame spacing types in order to decide the priority level. The SIFS is reserved 
for control packets (e.g Request to Send (RTS), Clear to Send (CTS), Acknowledgement (ACK), etc.). These packets have the highest priority and nodes can send packets immediately after the SIFS. The IEEE 802.11 standard gives the PCF mode a higher priority in comparison to the DCF mode. After PIFS, the base station can send scheduling time to nodes in the network without collisions. After SIFS and PIFS expire, all nodes wait till the end of DIFS and start their contending period by choosing a back-off timer in a contention window. The back-off timer is a random number between $\left[0, C W_{\min }\right]$ (the minimal value of contention window). The node which has the shortest back-off timer wins the channel and starts to transmit. All the others have to wait till the end of the transmission. In order to ensure the fairness, all sensors contend the channel after DIFS with the same value of initial contention window. Hence, they have the same probability to access the channel. To prevent a node from occupying the channel for a long time, after transmission of each packet, the node has to release the channel to other nodes. All nodes start to contend the channel again.

\subsection{A simple scenario}

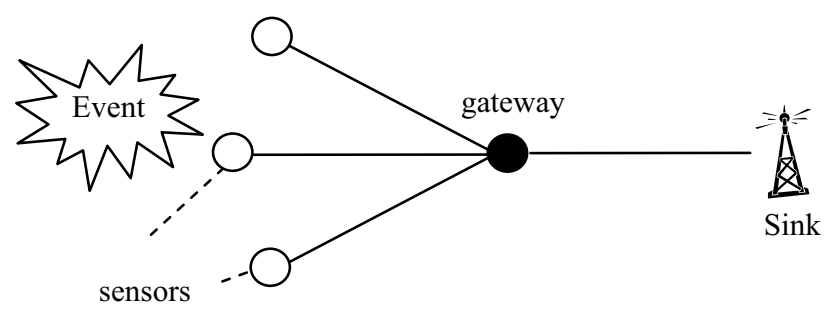

Fig. 2. A simple scenario of an event-driven WSN

Fig. 2 illustrates a simple scenario of an event-driven WSN. In this example, there are four sensors deployed in the network to detect an event. One sensor (the black one) has also the role of gateway to route packets to the sink for other sensors. The hidden terminal problem [6] prevents the network from simultaneous transmissions. If two or more nodes access the channel at the same time, there will be collisions. When an event happens, three sensors detect the event and send this event to the sink via the gateway node. In all existing MAC protocols, to guarantee the fairness in the network, every sensor accesses the channel with the same probability. Hence, we cannot know the transmission order of nodes in the network.

As nodes access the channel randomly, we can have different transmission scenarios. Fig. 3 illustrates a pessimistic and an optimistic transmission timeline of the network topology in Fig.2. Each transmission between two nodes takes $\Delta t$ time. As an event can be composed of many packets: the temperature of the last 10 minutes, a picture of a particular area of a machine, a sound etc, we refer the latency as the elapsed time between the moment that the event happens and the moment that the sink receives all packets of an event. To simplify the illustration, we suppose that each event is composed of two packets. 


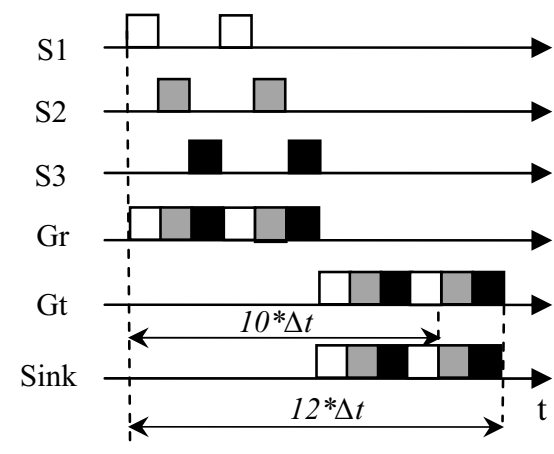

Gr : Gateway Receiver

(a) Pessimistic

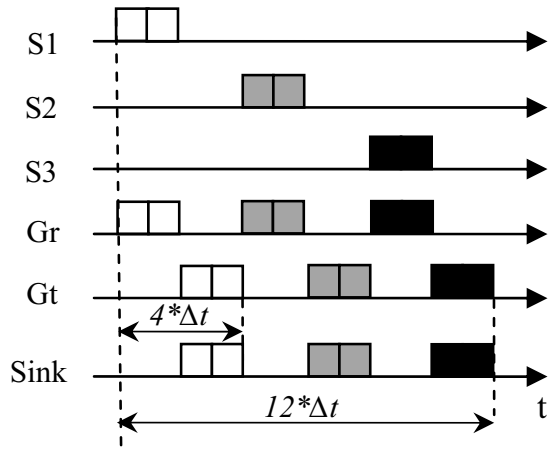

Gt : Gateway Transmitter

(b) Optimistic

Fig. 3. Transmissions timeline

In the pessimistic scenario (Fig. 3a), as the gateway node is a normal node and it has the same probability to access the channel, it has to wait until all sensors from S1 to S3 finish their transmission. Packets are buffered at the gateway before being transmitted to the sink. Hence, when an event happens, the sink receives the first event after $10^{*} \Delta t$ time. Then, the gateway continues to send packets to the sink and the sink receives all messages of the event after $12 * \Delta t$ time.

In the optimistic scenario (Fig. 3b), we have two remarks. First, after sending a packet, the winner sensor continues to occupy the channel and transmits its packet corresponding to the event. It does not release the channel to the others. Second, after receiving a packet from a sensor, the gateway node wins the channel and forwards the packet immediately to the sink. When an event happens, the sink receives the first event after $4^{*} \Delta t$ time. Then, the sink receives all messages related to the event after $12 * \Delta t$ time

As stated earlier, sensors are often spatially correlated and we do not need to receive all messages from all sensors in order to know about the event. The latency of the first $\mathrm{R}(R<N)$ events is the more important. In the optimistic scenario, the sink receives the first event after $4^{*} \Delta t$ while in the pessimistic scenario, the sink receives the first message after $10^{*} \Delta t$ which is much longer. That is why we call the scenario in Fig. $3 b$ an optimistic scenario.

These timelines illustrate just a simple scenario with a two-hop data transmission and two packets for each event. In general cases, the network topology is much larger which implies multi-hop data transmissions. An event can include many packets, it can be a picture taken by an image sensor or a sound recorded by a sound sensor. In these cases, the latency of the optimistic is much lower than the pessimistic scenario.

In all existing MAC protocols, and particularly in IEEE 802.11, nodes access the channel with the same probability. Therefore, the group of three sensors (S1, S2, and S3) has more probability to access the channel than the gateway (three times higher following the theory of probability). The probability that the pessimistic scenario happens is much higher than that of the optimistic scenario. We state that fairness does not optimize the transmission latency, particularly in multi-hop WSN. As we 
want to reduce the transmission latency, we need to find a method to guarantee that the transmission scenario would follow the optimistic scenario.

\section{LLMAC - a low latency MAC protocol for WSN}

In this section, we describe our proposal LLMAC which enhances the transmission latency for event-driven WSN. We start by introducing several hypotheses of our working context:

- First, we assume an event-driven WSN to detect certain events in a critical application where the latency is unacceptable.

- Second, each event is composed of a high number of packets: the temperature of the last 10 minutes, a picture, a sound etc.

- Third, we deploy a large WSN where sensors can be organized in a tree-based topology. Hence, when an event happens, sensors send data to the sink via gateway nodes in the data gathering tree [14].

- Finally, sensors are spatially correlated which implies the data redundant transmission. When an event occurs and $\mathrm{N}$ nodes detect the event, the sink only needs to receive information from $\mathrm{R}$ nodes $(R<N)$ in order to have enough information for the event to take a reaction. Hence, the objective of an event-driven WSN is to obtain information from R nodes within the shortest delay.

Starting from these hypotheses, we will present our proposition LLMAC, where we guarantee that the optimistic scenario always happens. We propose two main contributions:

- A change of the transmission policy from frame level to event level

- A new inter-frame spacing value in order to favor the data transmission of gateway nodes

\subsection{Rafale of frame}

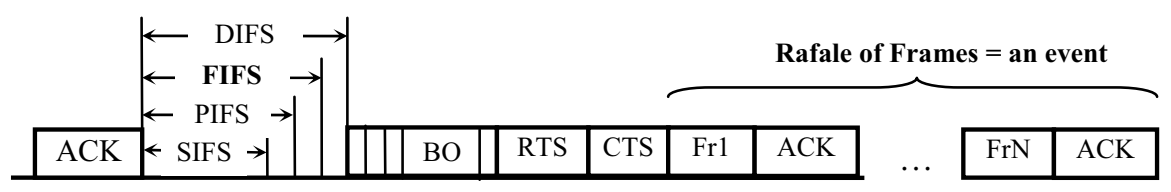

FIFS: Forward Inter-frame Spacing

Fig. 4. Enhancement of IEEE 802.11

In normal case, after each frame transmission, nodes have to release the channel and re-contend for another channel access. This prevents the case where a node abuse to occupy the channel all the time. Besides, this method also helps other nodes to have a chance to access the channel. However, this technique is not well adaptable to eventdriven where we are interested in the latency of an event, but not that of a frame. Therefore, we change the transmission policy from frame level to event level. 
Policy 1: After winning the channel, a node continues to transmit its packets until all packets concerning the event are completely sent.

In our proposal, we want nodes to complete their transmission for each event before leaving the channel for the others. Hence, in Fig. 4, in place of releasing the channel after each frame transmission, the winner node occupies the channel to send a rafale of frames. This rafale of frames can be a combination of sensed information: temperature, vibration, picture etc. The length of the rafale of frame is equal to the length of an event. In fact, by using a rafale transmission, other nodes lose the right to transmit and we do not guarantee the fairness of the system. However, we see a sensor network as a network with sensors which cooperate to achieve the same goal but not as a network where sensors compete to access the channel with different goals.

As a node can occupy the channel during the transmission of an event, it is not intercepted by other transmission and the latency for an event transmission is reduced. Here, we really make a trade-off between fairness and latency of the event. However, in event-driven WSN, as a short latency is crucial, it is preferable to scarify the fairness in order to obtain better latency results.

\subsection{Forward Inter-frame Spacing}

By using a rafale of frame transmission, we can guarantee a short delay transmission for each event in one-hop. However, an event-driven WSN is often large with multihop transmissions. Moreover, we have shown in the previous section that nodes use the same inter-frame spacing value (DIFS) and the same back-off value to guarantee the fairness. However, the fairness often makes data to be blocked at the gateway node, which increases the transmission latency. Therefore, we need to assure that the forwarding node can win the channel immediately after receiving a packet. Then, data will be forwarded immediately and will not be blocked at the gateway node. Here, we define a new inter-frame spacing that we call FIFS (Forward Inter-frame Spacing) (Fig. 4). This inter-frame spacing is shorter than DIFS and longer than SIFS and PIFS.

Policy 2: After receiving a packet, a forwarding node accesses the channel immediately with the Forward Inter-frame Spacing.

We know that inter-frame spacing defines priority level for channel access. By setting the FIFS shorter than DIFS, we give the priority to the transmission of nodes which use the FIFS. As forwarding nodes use this inter-frame spacing, they can access the channel before the contending period of normal nodes. After receiving a rafale of frames for an event, the forwarding node sets its inter-frame spacing to FIFS. Hence, once the FIFS expires, it starts to forward the rafale of frame immediately. This avoids the case where data is blocked at the gateway. The event can be sent multi-hop to the sink within the shortest delay.

\section{Performance Evaluation}

In this section, we prove the effectiveness of our proposal LLMAC in comparison to IEEE 802.11. As we refer to an event-driven WSN, the most important criterion that we are interested in is the latency of the event. 


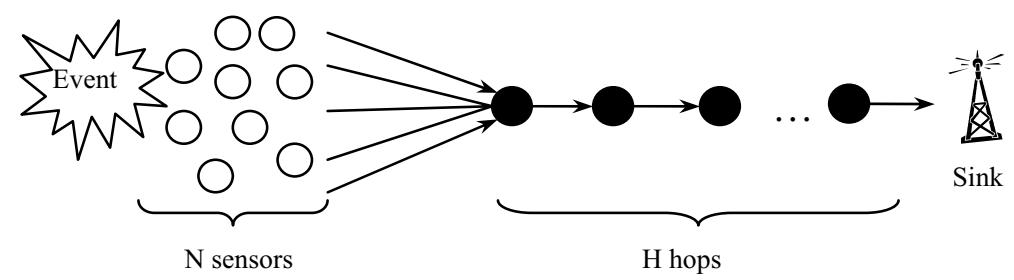

Fig. 5. Simulation topology

We simulate a multi-hop event-driven WSN with the topology described in Fig. 5. We use the OMNet++ simulator [15] to validate LLMAC. OMNet++ is a publicsource, component-based, modular and open-architecture simulation environment. When an event happens, $\mathrm{N}$ sensors can detect the event and send it to the sink via $\mathrm{H}$ hops. Each event is composed of $\mathrm{P}$ packets. Each packet has a size equal to the maximal size of an 802.11 frame. All other MAC parameters are set to the standard 802.11. The value of FIFS is set to be an average value between SIFS and DIFS. The objective of an event-driven WSN is to receive $\mathrm{R}(R<N)$ events within the shortest delay.

\subsection{Variation of $\mathbf{R}$}

In this simulation, we measure the transmission latency of R events. We set a WSN with these parameters: $N=4, H=2, P=10$. We vary the R value from 1 to $\mathrm{N}$ in order to know the latency of each event.

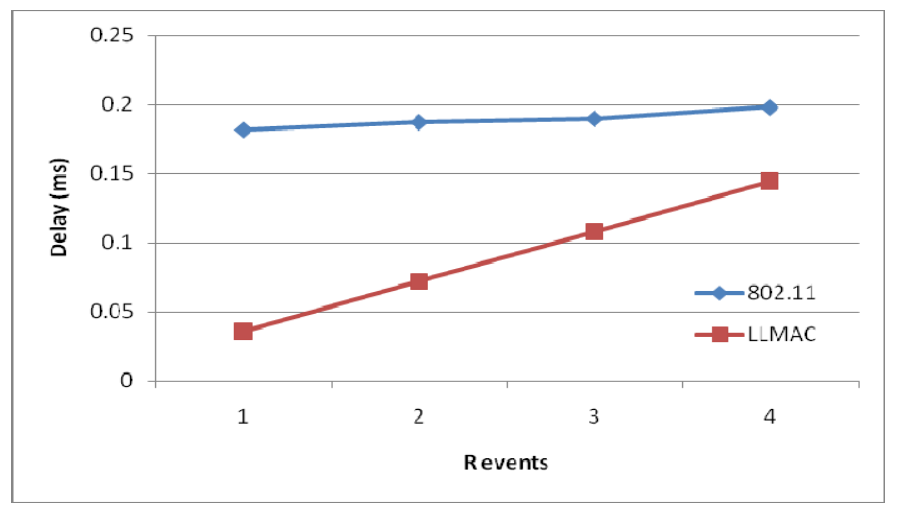

Fig. 6. Latency by varying $R$

Fig. 6 illustrates the transmission latency of the first R events. There are just four detected events by four nodes. Our proposal always guarantees the shorter delay in comparison to 802.11 in every case. With small $\mathrm{R}$ value, LLMAC performs better results in comparison to 802.11 because LLMAC favors the transmission of each event in multi-hop while 802.11 favors the transmission of each packet in one-hop. As 
$\mathrm{R}$ increases, the transmission delay of LLMAC is slightly increased. However, even in the case when all events arrive at the sink, LLMAC always performs a better result than 802.11 because LLMAC uses only one RTS/CTS exchange for all transmission of one event. Between each packet transmission of an event, LLMAC does not use RTS/CTS.

\subsection{Variation of $P$}

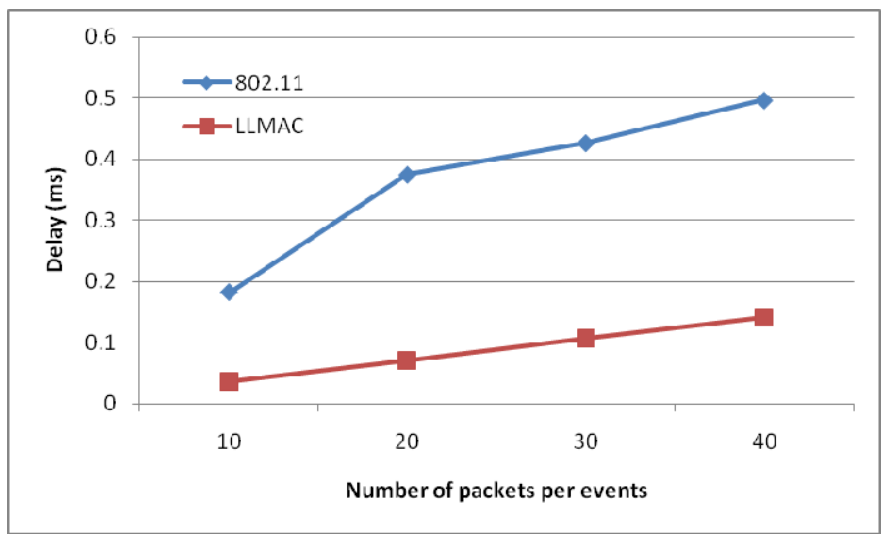

Fig. 7. Latency by varying $P$

As mentioned in the previous section, our proposal is aimed to applications where an event is composed of many packets. In this simulation, we want to show the effect of changing the number of packets per event in our proposal and 802.11. We consider a WSN with $N=4, H=2, R=1$. We vary the P value from 10 to 40 packets. In all case, we see that LLMAC always performs a better result than 802.11. When we increase the number of packets for each event, the latency of LLMAC is slightly increased. However, the latency of 802.11 is increased much faster.

\section{CONCLUSION AND FUTURE WORKS}

In this paper, we have presented and analyzed an enhancement of 802.11 which we call LLMAC, a low latency MAC protocol for event-driven wireless sensor network. In this type of WSN, the latency is the most important criterion which decides on the effectiveness of the system. Our method makes a trade-off between fairness and latency in order to offer a shorter latency. By simulation evaluations, we have proved that our proposal clearly improves the latency transmission in comparison to 802.11.

Most of existing works for MAC protocol in WSN often propose a sleep mode for sensors in order to save energy. In sleep mode, nodes cannot sense or transmit information. The latency would be very high in this type of MAC protocols. 
Therefore, they are more suitable for monitoring application but not for event-driven WSN. Our proposal does not take the energy consumption into account. In the future, we would like to modify the LLMAC with energy consumption awareness. Moreover, we will try to evaluate our proposal in a real testbed framework in the near future.

\section{Acknowledgements}

We would like to express our thanks to the Franche Comté region for their financial support for our research at the Franche Comté University, Besançon, FRANCE.

\section{References}

1. Specification of the Bluetooth System: Core. [Online]. Available: http://www.bluetooth.org/, 2001.

2. V. Rajendran, K. Obraczka, J.J. Garcia-Luna-Aceves, "Energy-Efficient, Collision-Free Medium Access Control for Wireless Sensor Networks", Proc. ACM SenSys 03, Pages: 181 - 192, Los Angeles, California, Nov. 2003.

3. W. R. Heinzelman, A. Chandrakasan, and H. Balakrishnan, "Energy efficient communication protocols for wireless microsensor networks," in Proc. Hawaii Int. Conf. Systems Sciences, pp. 3005-3014, Jan. 2000.

4. Wireless LAN Medium Access Control (MAC) and Physical Layer (PHY) Specification, IEEE Std. 802.11-1999 edition.

5. W. Ye, J. Heidemann, D. Estrin, "Medium Access Control With Coordinated Adaptive Sleeping for Wireless Sensor Networks", IEEE/ACM Transactions on Networking, Volume: 12, Issue: 3, pp. 493 - 506, June 2004.

6. A Tanenbaum, "Computer Networks", 4th edition, Prentice Hall, Pearson Education 2003.

7. T. van Dam and K. Langendoen, "An Adaptive Energy-Efficient MAC Protocol for Wireless Sensor Networks," in ACM SenSys 2003, Nov. 2003.

8. Polastre, J., Hill, J., Culler, D. "Versatile Low Power Media Access for Wireless Sensor Networks", in Proc. of the ACM SenSys Conf., Baltimore, pp. 95-107, 2004.

9. K. Jamieson, H. Balakrishnan, and Y. C. Tay. "Sift: A MAC protocol for event-driven wireless sensor networks". Third European Workshop on Wireless Sensor Networks (EWSN), Feb. 2006

10. Y.C. Tay, K.Jamieson, H. Balakrishnan, "Collision-minimizing CSMA and Its Applications to Wireless Sensor Networks", IEEE Journal on Selected Areas in Communications, Volume: 22, Issue: 6, pp. 1048 - 1057, Aug. 2004.

11. S. Blake, D. Black, M. Carlson, E. Davies, Z. Wang, and W. Weiss. An Architecture for Differentiated Services, Dec. 1998.

12. M. Benveniste, G. Chesson, M. Hoehen, A. Singla, H. Teunissen, and M. Wentink. EDCF Proposed Draft Text. IEEE working document 802.11-01/131r1, Mars 2001.

13. L. Romdhani, Q. Ni, and T. Turletti. "AEDCF: Enhanced Service Differentiation for IEEE 802.11 Wireless Ad-hoc Networks", pp. 1373-1378 In the proceeding of the IEEE Wireless Communications and Networking Conference WCNC03, New Orleans, Louisiana, USA, Mars 2003.

14. B. Krishnamachari, D. Estrin and S. Wicker, "The impact of data aggregation in wireless sensor networks", in International Workshop on Distributed Event-based Systems, 2002

15. OMNet++ Community Site. [Online] Available http://www.omnetpp.org/ 